\title{
Applying Frame Theory to Psychiatric Classification
}

\author{
Jürgen Zielasek, Gottfried Vosgerau, Wolfgang Gaebel, \\ Karin Fauerbach, Irem Girgin, Sarah Jungbluth, Julia \\ Weiland \& Sebastian Löbner
}

\begin{abstract}
We applied Barsalou's Frame Theory to analyze the structure of operationalized classificatory texts used in psychiatry, such as the International Classification of Disorders, Chapter V (ICD-10), and the Diagnostic and Statistical Manual (DSM-IV), which provide the basic terminology for today's psychiatric classification system. We studied the classificatory principles of "schizophrenia" and "specific phobias" as examples of mental disorders. In addition, we studied Parkinson's disease as an example of a disorder on the border between neurology and psychiatry. Initial results suggest that although the texts of modern classification systems are highly operationalized and appear straightforward and simple, their internal structure is highly complex with subframe structures of divergent types emerging. Also, the comparison of both modern systems of classification shows that the differences are greater than just time-course differences or terminological differences. We show that by applying Barsalou's frame theory, internal structures of standard classificatory texts in psychiatry may become discernible, and that focusing on well-defined concepts of brain functions like the sense of agency promises to yield useful insights into the pathophysiology, symptomatology and classifications of major mental disorders.
\end{abstract}

\section{Introduction}

Barsalou developed a theory of frames as the general format of the representation of concepts in human cognition (Barsalou 1992a, 1992b). The theory was further developed, and integrated into Barsalou's theory of conceptual symbol 
systems (Barsalou 1999). Barsalou frames represent their referents by means of a recursive structure in terms of attributes and the values they take. Following the conventions developed in Petersen (2007/2015), Barsalou frames can be represented by directed graphs. ${ }^{1}$ In a frame for an arbitrary category of objects, a central node represents the object itself; it is marked with a double line. The node is "central" in that all arrows originate directly from it or from nodes connected to it. Labeled arrows connect a node to other nodes. The arrows represent attributes, the labels name them. The nodes to which the arrows lead represent the values that the attributes take. The number of attributes is not limited, nor is the depth of embedding.

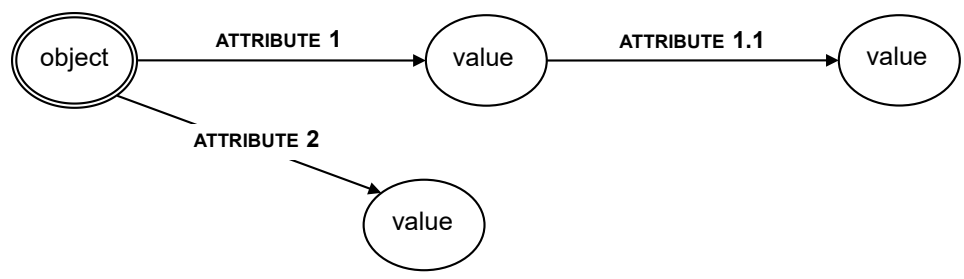

Figure 1: General structure of a Barsalou frame

Crucially, the attributes are functional relations: for any object they assign exactly one value and for any object the same attribute can only be applied once. In frame graphs it is not permissible to have more than one arrow with the same label originate from one and the same node. Different nodes may, however, have the same attribute. Think, for example, of a frame-format physical description of a person. Possible attributes can include Height, Weight, and AGE. ${ }^{2}$ Any person, at a given time, has the descriptive dimensions of height, weight or age only once and each of these take just one value. For a simple notation for the fact that for an object $x$ the attribute a takes the value $y$, one can use the usual mathematical notation for a function taking a value for a given argument: " $a(x)=y$ ”. One can write "HEIGHT(Jones) $=1.76 \mathrm{~m}$ " for the fact that the attribute HEIGHT for the person Jones takes the value $1.76 \mathrm{~m}$. A nonfunctional attribute would be a characteristic such as a 'disease': namely, a person can have several, just one or no

\footnotetext{
1 Barsalou (1992a, 1992b) uses directed labeled graphs, too. However, his representations have a more complex, although essentially equivalent, structure. See Petersen (2007/2015) for a discussion of the alternative graph representations of frames.

2 We use small capitals for attribute terms.
} 
diseases at all. Consequently, the attribute "disease" would not necessarily take a unique value. It would be inappropriate to notate the fact that Jones suffers from diabetes as "DISEASE(Jones) = diabetes"; if Jones suffered from a bronchitis at the same time, one would have to put this as "DISEASE(Jones) = bronchitis". However, if DISEASE really were to be a function(al attribute), its value for a given person at a given time cannot be both diabetes and bronchitis because these are different diseases. In constructing frames for the representation of concepts, attributes have therefore to be chosen very carefully to use only attributes that are really functions. Representing the fact that a person suffers from diabetes would require a frame with attributes such as INSULIN PRODUCTION or INSULIN RESISTANCE.

Frame-format descriptions are thus descriptions in terms of functional attributes and the values they take. Such descriptions are very frequently used in practice in one form or the other. For example, a lab report with values for various substances in the blood such as Fe, $\mathrm{Mg}$, or cholesterol, represents a frame in terms of concentrations of these substances in the blood sample analyzed. A reference to a publication represents a frame-format description in terms of author(s), year of publication, title, publisher, etc. Another instance of frame-format description is passports; they contain a frame description of the bearer.

Barsalou frames may also contain various types of constraints that relate to the values of attributes. For example, a constraint might delimit the range of possible values for an attribute, or it might correlate the values of two attributes.

Frames for diseases or disorders would start from a frame for the person with attributes added for those parts or functions of the organism which are affected. A disease can be described at different levels. A description of diabetes at the level of diagnostics would refer to attributes such as blood sugar level, while a description at the organic level might relate to the function of the pancreas. Capturing the causes of the disease would require attributes relating to lifestyle or to the genetics of the person. The description of a disease would then essentially involve the choice of relevant attributes and the values they take. Such a frame may be restricted to a certain level of description or it may combine more than one level. Including both the organic and the symptomatic level would enable one to represent causal relations between elements of the frames, for example between insulin production and blood sugar.

Using frames for the analysis of mental disorders is demanding, but offers the opportunity for a more precise and explicit conceptual analysis. The available 
descriptions of disorders do not come in terms of functional attributes (see Tables 1 and 2 below). To begin with, in most cases of particular mental disorders it is not made explicit what the respective disorder is a disorder of. What exactly is disturbed in schizophrenia? Is there a uniform disorder underlying all those cases currently classified as a particular disorder? The usual descriptions of mental disorders describe symptoms and diagnostics, such has "hearing voices" in the case of schizophrenia. The description of a disorder may refer to behavioral characteristics or to other levels such as bodily symptoms or genetics. It may be in terms of phenomena or in terms of malfunctioning. It will include temporal characteristics such as the frequency and duration of symptoms, the age of the patient, the chronological progression of the disorder. It may also include causal components such as lifestyle, social environment, infections, intoxication, etc. It must provide for a weighting of symptoms, for exclusion criteria, and for provisions such that a certain number out of all possible symptoms must be present. A frame approach should first make a decision about the level(s) of description which it aims at and will then have to try to determine the appropriate set of attributes for capturing the characteristics of the disorder. In this article, we will represent initial exploratory heuristic frame descriptions of three disorders: schizophrenia, specific phobias, and Parkinson's disease. The last section represents considerations towards a more principled approach to the frame analysis of mental disorders in terms of faculties of the mind.

\section{Classification of mental disorders}

Two major classification systems for mental disorders have been developed and are in use globally: the World Health Organization (WHO) publishes the "International Classification of Disorders," which is currently in its 10th revision (hence "ICD-10") (World Health Organization, 1990) and the American Psychiatric Association (APA) publishes the "Diagnostic and Statistical Manual," which is currently available in its fourth edition (hence "DSM-IV") (American Psychiatric Association, 2000). Both systems are currently being revised by international working groups with a view to publish the newly revised versions ICD-11 and DSM-V in 2013/2014. Both classification systems share a number of features:

- They are operationalized: they explicitly specify rules on how to arrive at a certain diagnosis 
- They are anosological: they do not make assumptions about the nosological entities that may underlie a certain diagnosis. This implies that they do not make assumptions about the pathophysiology of any of the mental disorders classified in the respective diagnostic system.

Both systems have been tested for inter-rater reliability in the early 1990's, and one of their strengths and advantages is clearly that they have reduced the ambiguities previously associated with psychiatric diagnostics. After appropriate training, the inter-rater reliability is around $80 \%$. These systems have become widely accepted internationally. The majority of international psychiatric scientific literature uses either ICD-10 or DSM-IV. A survey of the use of ICD or DSM in psychiatric journals showed that some country-specific preferences are still observable between both systems, i. e., US-psychiatrists prefer DSM-IV, whereas German psychiatrists prefer ICD-10 (Lopez-Munoz et al., 2008).

As a rule, the constitutive features of mental disorder specified in these classifications include i) a syndrome of clinical symptoms, ii) some disease course characteristics and iii) the results of laboratory tests or neuroimaging methods. The latter, however, are mainly used for detecting brain tumors, inflammatory or metabolic disorders as causes of mental disorders.

In Germany, the use of the ICD-10 has been mandatory since 2000 for in- and out-patient services. For example, reimbursement by health insurance companies in the somatic disorders is based on diagnosis-related groups, which are founded in ICD-10 diagnoses. The official version is published by the German Institute of Medical Documentation and Statistics (DIMDI), with annual updates of the German modification (ICD-10-GM), so that the actual 2010 version of the ICD10-GM features some minor differences compared to the original WHO ICD-10 version (www.dimdi.de). The German-language version issued by DIMDI is a trinational version which is also valid in Austria and Switzerland.

ICD-10 contains all somatic and all mental disorders, the latter are listed in Chapter $\mathrm{F}$ and contain the following major diagnostic groups (Table 1).

While the somatic disorders are only listed in ICD-10 and no specification for their diagnosis is given, the mental disorders are explicitly defined considering characteristic symptoms, time-course criteria, and exclusion criteria. In contrast to ICD-10, DSM-IV only deals with mental disorders, but also contains explicit rules for arriving at certain diagnoses similar to chapter F of ICD-10. A number of differences still exist between the diagnostic rules for some - but not all - 
Table 1: Mental Disorders in ICD-10 (Chapter F) (WHO, 1992)

\begin{tabular}{|c|l|}
\hline Chapter F class & Designation \\
\hline F0 & Organic, including symptomatic mental disorders \\
\hline F1 & Mental and behavioral disorders due to psychoactive substance use \\
\hline F2 & Schizophrenia, schizotypal and delusional disorders \\
\hline F3 & Mood [affective] disorders \\
\hline F4 & Neurotic, stress-related and somatoform disorders \\
\hline F5 & $\begin{array}{l}\text { Behavioral syndromes associated with physiological disturbances and phys- } \\
\text { ical factors }\end{array}$ \\
\hline F6 & Disorders of adult personality and behavior \\
\hline F7 & Mental retardation \\
\hline F8 & Disorders of psychological development \\
\hline F9 & $\begin{array}{l}\text { Behavioral and emotional disorders with onset usually in childhood and } \\
\text { adolescence }\end{array}$ \\
\hline
\end{tabular}

mental disorders between both classification systems, and those pertaining to the mental disorders discussed in this chapter will be delineated in the ensuing text. It should be mentioned at this point that there are also a number of regional variations of classification systems still in use for mental disorders, e.g., in China or Latin America, but in international research and for the purposes of international scientific projects, such regional codes have not gained any importance.

Besides the advantages of providing highly reliable, standardized and internationally widely accepted rules for diagnosing mental disorders, these classification systems have also been criticized for the aforementioned differences in operational details, for not including pathophysiological processes, and for agglomerating a range of putatively different nosological entities into single disease groups. These issues are currently being debated intensively in the APA and WHO workgroups in charge of revising DSM-IV and ICD-10. However, even considering the tremendous progress in elucidating pathophysiological processes involved in a range of mental disorders, it currently appears rather unlikely that these advances have already been made far enough to warrant the inclusion of pathophysiology criteria in DSM-V or ICD-11. 


\section{Applying frame theory to texts of psychiatric classification}

The main purpose of this initial stage of a joint research project between the Department of Psychiatry and Psychotherapy and the Department of Linguistics and Information Science of the Heinrich-Heine-University Düsseldorf was to demonstrate the feasibility of using Barsalou's Frame Theory to analyze texts of psychiatric classification with a view to systematically assessing the structure underlying the classification of mental disorders. The selection of mental disorders to be studied in the first phase of the research project was guided by the idea that one of the more frequent mental disorders was to be included. It should be diagnosed on the basis of clinical symptoms and it should provide some variations of time-course. We chose schizophrenia for this purpose, as it provides a large number of cases and fulfills these criteria. It has a range of time-course variants, and it is characterized by a set of hierarchical clinical diagnostic criteria. A second mental disorder was studied because in the course of analyzing schizophrenia, we realized that the analysis was hampered by a lack of information about the neurobiological foundations of schizophrenia, and the frame structure turned out to indicate a high degree of complexity. We therefore chose the group of specific phobias, since the neurobiology of fear disorders is comparatively better known, and the clinical courses and characteristics are not as complicated as in schizophrenia. We decided to round up this initial set of investigations with a neurological disorder featuring a clearly defined neurobiological foundation and associated symptoms of a mental disorder: Parkinson's disease is characterized by the degeneration of a set of neurons in the midbrain and ensuing degeneration of the fiber tracts that connect these specific areas of the midbrain with other brain areas. This results in the typical shaking palsy of patients with Parkinson's disease. In addition, approximately $30 \%$ of all patients with Parkinson's disease also suffer from affective or cognitive symptoms, which may lead to mental disorders such as depression and dementia.

\subsection{Frame analysis of schizophrenia}

Both DSM-IV-TR (DSM-IV Text Revision) and ICD-10 feature a range of schizophrenia-like symptoms of importance for diagnosing schizophrenia and schizophrenia-related disorders like persistent delusional syndromes or schizoaffective 
disorder, which constitutes an overlap of both mood symptoms and schizophrenia-like symptoms. For the purpose of the initial analysis of these criteria, our project focused on the major groups of "schizophrenia" (F20 in ICD-10; 295.1090 in DSM-IV). The diagnosis of schizophrenia according to DSM-IV or ICD-10 is a multi-step process. First, it is important for the clinician to ascertain that a number of symptoms are present for a sufficiently prolonged period of time. While the types of symptoms are compatible - but not identical - between both diagnostic systems, the time course criterion varies significantly (four weeks in ICD-10 and six months in DSM-IV). The symptoms comprise so-called "positive" symptoms like hallucinations and delusions and "negative" symptoms like loss of interest, lack of initiative and social withdrawal. Table 2 gives an overview of the diagnostic criteria comparing DSM-IV (text revision edition of 2000, hence "DSM-IV-TR") and ICD-10:

It is important to realize that two partly divergent sets of diagnostic categories exist. However, the principal components are comparable. We chose to use the ICD-10 criteria for a first analysis, since it is the type of criteria set relevant for Germany, and since it is widely used in Europe as compared to the DSMIV criteria. Figure 2 shows some aspects that a frame for the ICD-10 definition of schizophrenia may contain.

This frame shows that one of the major challenges will be to determine which functions underlie the pathophysiology of the major symptoms like hallucinations and delusions. We have added place holders, because much more work is needed to demonstrate whether functional systems such as a "reality check" system exist in the human brain, and whether they become dysfunctional in schizophrenia. We are currently performing such investigations by deriving information from the scientific literature on the set of such systems as identified by neuroimaging or other assessment techniques. It is likely that such systems, or "modules" as they are called in systems analyses of the central nervous system, are at the root of the pathophysiology of the functional impairments observed in mental disorders (Zielasek \& Gaebel, 2008; Seitz et al., 2011). Also, this initial analysis shows that the structure of the schizophrenia frame will be very complicated, probably leading to a substantial number of subframes. As there are associations between the positive symptoms as a group of symptoms, this may lead to a formal constraint (for example between the various types of delusions differentiated in the ICD-10 criteria). In the course of developing DSM-V, the 


\section{Applying Frame Theory to Psychiatric Classification}

Table 2: Operationalized diagnostic criteria of schizophrenia compared between ICD-10 (WHO, 1992) and DSM-IV-TR (American Psychiatric Association, 2000).

\begin{tabular}{|l|}
\hline ICD-10 \\
\hline Clinical criteria \\
\hline $\begin{array}{l}\text { A) Thought echo, thought insertion or withdrawal, and } \\
\text { thought broadcasting }\end{array}$ \\
\hline $\begin{array}{l}\text { B) Delusions of control, influence of passivity; delusional } \\
\text { perception }\end{array}$ \\
\hline $\begin{array}{l}\text { C) Hallucinatory voices giving running commentary on the } \\
\text { patient's behavior, or discussing the patient among them- } \\
\text { selves, or other types of hallucinatory voices coming from } \\
\text { some part of the body }\end{array}$ \\
\hline
\end{tabular}

DSM-IV-TR

Criterion $\mathrm{A}$

1) Delusions

2) Hallucinations

some part of the body

D) Persistent delusions of other kinds that are culturally inappropriate and completely impossible

E) Persistent hallucinations in any modality when accompanied either by fleeting of half-formed delusions without clear affective content, or by persistent over-valued ideas, or when occurring every day for weeks or months on end

F) Breaks or interpolations in the trains of thought, resulting in incoherence or irrelevant speech, or neologisms

\begin{tabular}{l}
\hline G) Catatonic behavior \\
\hline H) Negative symptoms \\
\hline
\end{tabular}

A significant and consistent change in the overall quality

of some aspects of personal behavior

\section{Evaluation criterion}

4) Grossly disorganized or catatonic behavior

5) Negative symptoms

A minimum of one very clear symptom (and usually two or more if less clear-cut) belonging to any one of the symptoms in groups (A) to (D) above, or symptoms from at least two of the groups referred to as $(\mathrm{E})$ to $(\mathrm{H})$

\section{3) Disorganized speech}

5) Negative symptoms

\begin{tabular}{|l|l|} 
& more voices conversing with each other \\
\hline & Criterion B \\
\hline Time criterion & Social/occupational dysfunction \\
\hline & Time criterion \\
\hline One month or more & Criterion C \\
\hline & $\begin{array}{l}\text { Duration: continuous signs of the disturbance persist for } \\
\text { at least } 6 \text { months. This } 6 \text { month period must include at } \\
\text { least one month of symptoms that meet Criterion A }\end{array}$ \\
\hline Exclusion criteria & Exclusion criteria \\
\hline $\begin{array}{l}\text { Extensive depressive or manic symptoms unless it is clear } \\
\text { that schizophrenic symptoms antedated the affective dis- } \\
\text { turbance }\end{array}$ & Criterion D \\
\hline & Schizoaffective and mood disorder exclusion \\
\hline & \\
\hline & Criterion E \\
\hline Presence of overt brain disease & Substance/general medical condition exclusion \\
\hline States of drug intoxication or withdrawal & \\
\hline & Criterion F \\
\hline & Relationship to a pervasive developmental disorder \\
\hline
\end{tabular}


search for such "clusters" of symptoms became very important. Nevertheless, the associations are merely statistical, since every positive symptom may occur independently of others. The same holds for the association between symptoms and course characteristics. Even the association between certain symptoms and prognosis is not a strict association, but rather a statistical association. This leads to the question as to how the different course specifiers may be incorporated into a frame analysis. Schizophrenia has different course types which are specified in DSM-IV as shown in Table 3.

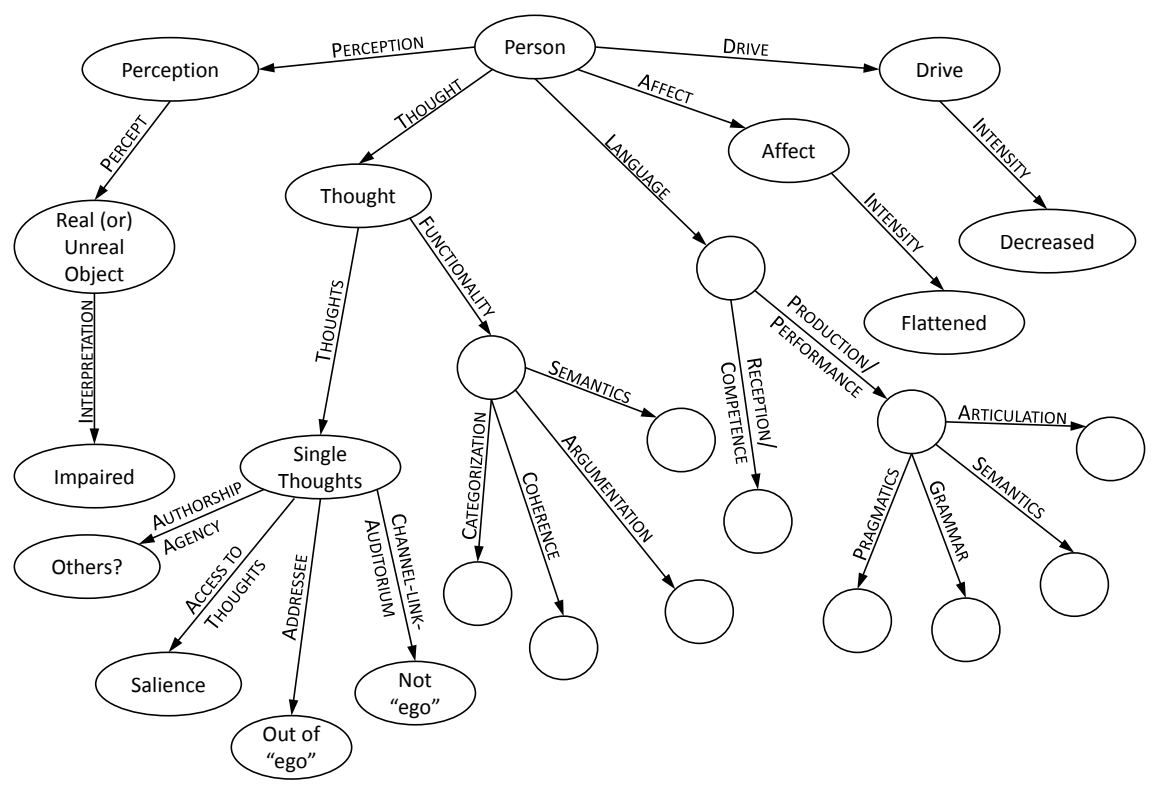

Figure 2: Some aspects of a schizophrenia frame analysis

It will be a major issue to devise methods of representing such temporal aspects of mental disorders in the usual notation of frames. This initial analysis is at best very preliminary and subject to change as research into the pathophysiology advances. However, schizophrenia does not lend itself easily to frame analysis, and may even indicate necessary extensions of frame theory once temporal and causal aspects come into play. Another challenge arises when sets of criteria with not yet clarified relations to functional systems of the brain are used. Although the latter is not necessary for establishing diagnostic categories with prognostic validity 
Table 3: Course specifiers of schizophrenia in DSM-IV-TR (American Psychiatric Association, 2000)

\begin{tabular}{|cl|}
\hline \multicolumn{1}{|c|}{ Description } \\
\hline 1. & Episodic with inter-episode residual symptoms \\
\hline 2. & Episodic with no inter-episode residual symptoms \\
\hline 3. & Continuous \\
\hline 4. & Single episode in partial remission \\
\hline 5. & Single episode in full remission \\
\hline 6. & Other or unspecified pattern \\
\hline
\end{tabular}

and for indicating the necessity of therapy, it would become a necessity if functional subsystems and their relationship to symptoms needed to be defined for frame analysis. With the current knowledge, frame analysis could still be useful for analyzing historic shifts in the definition and understanding of "schizophrenia" as a diagnostic construct in psychiatry - not relying on the identification of the "true" modules disturbed in schizophrenia, but rather on terminological descriptions. This will be the focus of the ongoing analysis besides the definition of the dysfunctional modules. Central texts for such an analysis would be the conception of "dementia praecox" by Kraepelin (1899), the initial definition of the schizophrenias as a group of mental disorders by Bleuler (1911), and the introduction of a hierarchy of schizophrenia-defining symptoms by Schneider (1971).

\subsection{Frame analysis of specific phobias}

While fear is a normal and physiological element of human existence serving to protect an individual in dangerous situations, its diagnostic value relates to inappropriate fears occurring in harmless situations, or with negative consequences for the individual in excess of any useful warning function of fear. Both ICD-10 and DSM-IV define a variety of fear disorders ranging from stimulusspecific anxiety disorders like the so-called "specific phobias" (including fear of narrow rooms, fear of spiders etc.) to generalized anxiety disorders. ICD-10 (http://www.who.int) defines specific phobias as "phobias restricted to highly specific situations such as proximity to particular animals, heights, thunder, darkness, flying, closed spaces, urinating or defecating in public toilets, eating certain foods, dentistry, or the sight of blood or injury." For the purpose of frame analysis, we chose specific phobias because in contrast to schizophrenia they are 
characterized by a rather narrow spectrum of symptoms, clearly defined fearinducing stimuli, and a rather well-known neurocircuitry. Thus, it could be hoped that frame analysis would lead to rather simple frame structures. However, neuroimaging frequently shows that phobic stimuli activate a range of neurocircuits (Pull, 2008; Damsa et al., 2008) including the amygdala and other structures of the brain like the anterior cingulate cortex and the insular cortex. Such "fear circuitries" are necessary conditions for bringing about fear, but it is still not completely known what exactly goes wrong with this circuitry in phobias. An intriguing question also arises as to the role of variations in genes involved in the metabolism of the neurotransmitter serotonin (Munafo et al., 2008), as these seem to increase the likelihood of the development of fear disorders. Also, learning experiences seem to play an important role. Thus, while it is quite well known which brain regions are involved and which normal functions are usually served by such regions, it is still a considerable step to fully explain the psychopathology, i. e., the set of signs and symptoms so characteristic of specific phobias. There are still missing links in the chain of events from genetic predisposition via learning experiences and initial fear reactions to the full clinical picture of a specific phobia. All of these steps would ideally be modeled in frame analyses of the future. A peculiar challenge lies in the delineation from "healthy" fear reactions. Similar to the dimensional assessment of hallucinations and delusions in schizophrenia, there appears to be a substantial overlap between fear reactions in the healthy control population and in those individuals who develop anxiety disorders. In addition, there is not only an emotional reaction to fear stimuli, but also a vegetative reaction with a diverse range of somatic signs like sweating and increased heart rates. Thus, a frame for specific phobias will need to include a range of functional circuits. An initial proposal is made in Figure 3.

\subsection{Frame analysis of Parkinson's disease}

Parkinson's disease used to be characterized as a neurodegenerative disorder with relatively specific degeneration of a set of neurons in the substantia nigra of the midbrain. The neurotransmitter produced by these neurons is dopamine, and consequently there is a loss of dopaminergic neurotransmission in the target projection area of these neurons in the striate corpus of the basal ganglia. This leads to the main symptoms of Parkinson's disease, i. e., resting tremor, akinesia, 


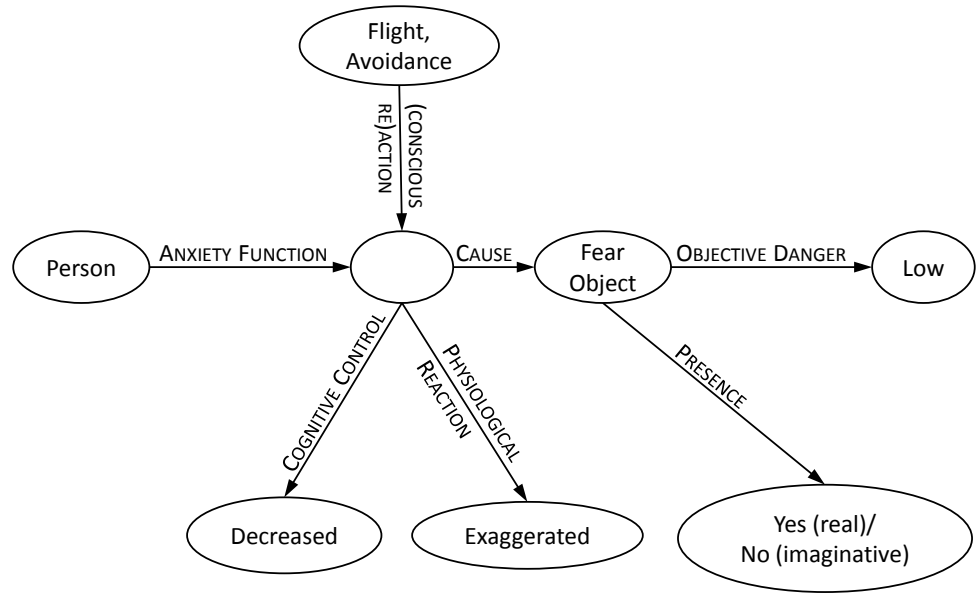

Figure 3: Aspects of a frame analysis of specific phobias

and rigor (Lees et al., 2009). However, other neurotransmitter systems are also involved in the neurodegenerative process. An intricate neurocircuitry of motor control underlies these pathophysiological processes.

A simple first approach towards a frame analysis of the impairment of the motor system in Parkinson's disease would result in a frame as depicted in Figure 4. This frame does not yet take into account the fact that a significant proportion of patients with Parkinson's disease not only suffer from motor symptoms, but also from affective and cognitive impairments. It is not completely understood how these are related to the primary nigrostriatal degenerative process, but may involve a spread of the initially locally confined neurodegenerative process to serotonergic and noradrenergic brain systems in depressive symptoms (Storch et al., 2008) and a more widespread involvement of cholinergic neurotransmission and frontostriatal circuits in patients with cognitive symptoms (Zgaljardic et al., 2004). A unifying hypothesis for the relationship between the loss of dopaminergic neurons and the ensuing neurocircuitry alterations leading to affective and cognitive symptoms has been proposed by Calabresi and colleagues (2006). Thus, similar to schizophrenia and specific phobias, the pathophysiology of mental disorders even in the framework of well-defined neurodegenerative processes involves a large number of brain areas and neurocircuits, making frame analysis 


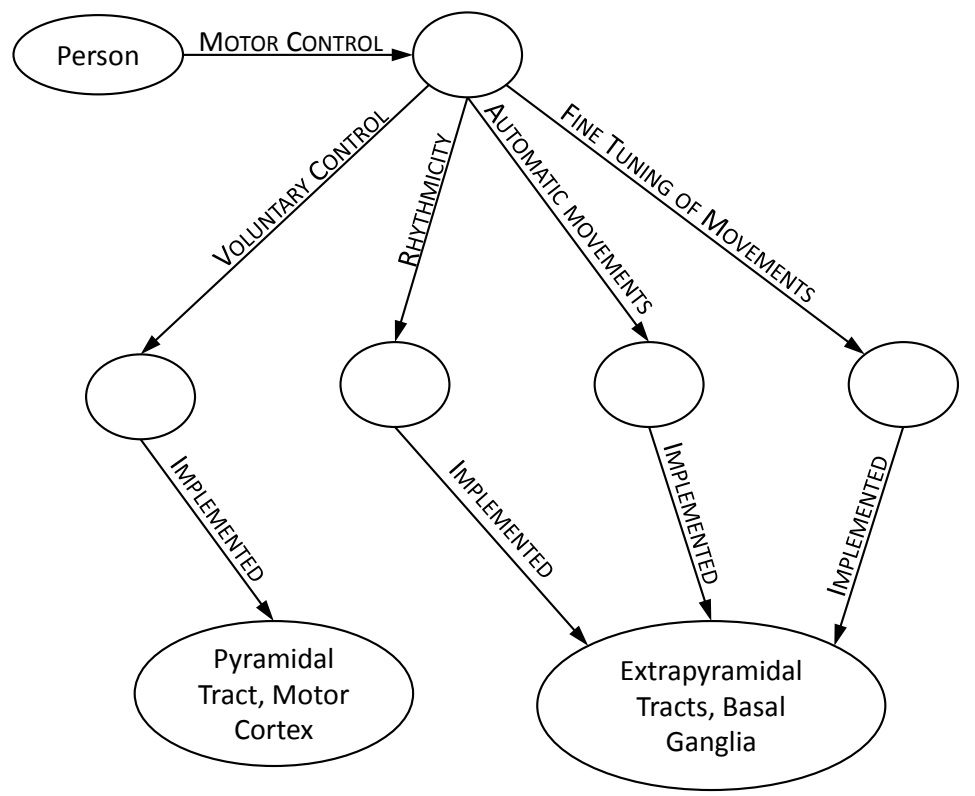

Figure 4: Frame of neuronal elements involved in motor symptoms in Parkinson's disease

difficult especially when higher brain functions involved in mood and cognition are affected.

\section{Frame analysis of the pathophysiology of mental disorders}

In order to gain a foothold in this complex area, we began an analysis of the concept of "agency," which is central to understanding many core features of mental disorders. We assume that the sense of agency is based on an integration process (weighting) of different agency cues which are provided by different processes (or "modules"), including motor control, perception, proprioception, thinking (background beliefs). Therefore, delusions of control cannot be explained by a breakdown in motor processes only - in contrast, we need a multifactorial account of agency that integrates the different agency cues mentioned above (Synofzik, Vosgerau \& Newen 2008). This account can thus serve as an example of the analysis of symptoms in terms of faculties of the mind. 


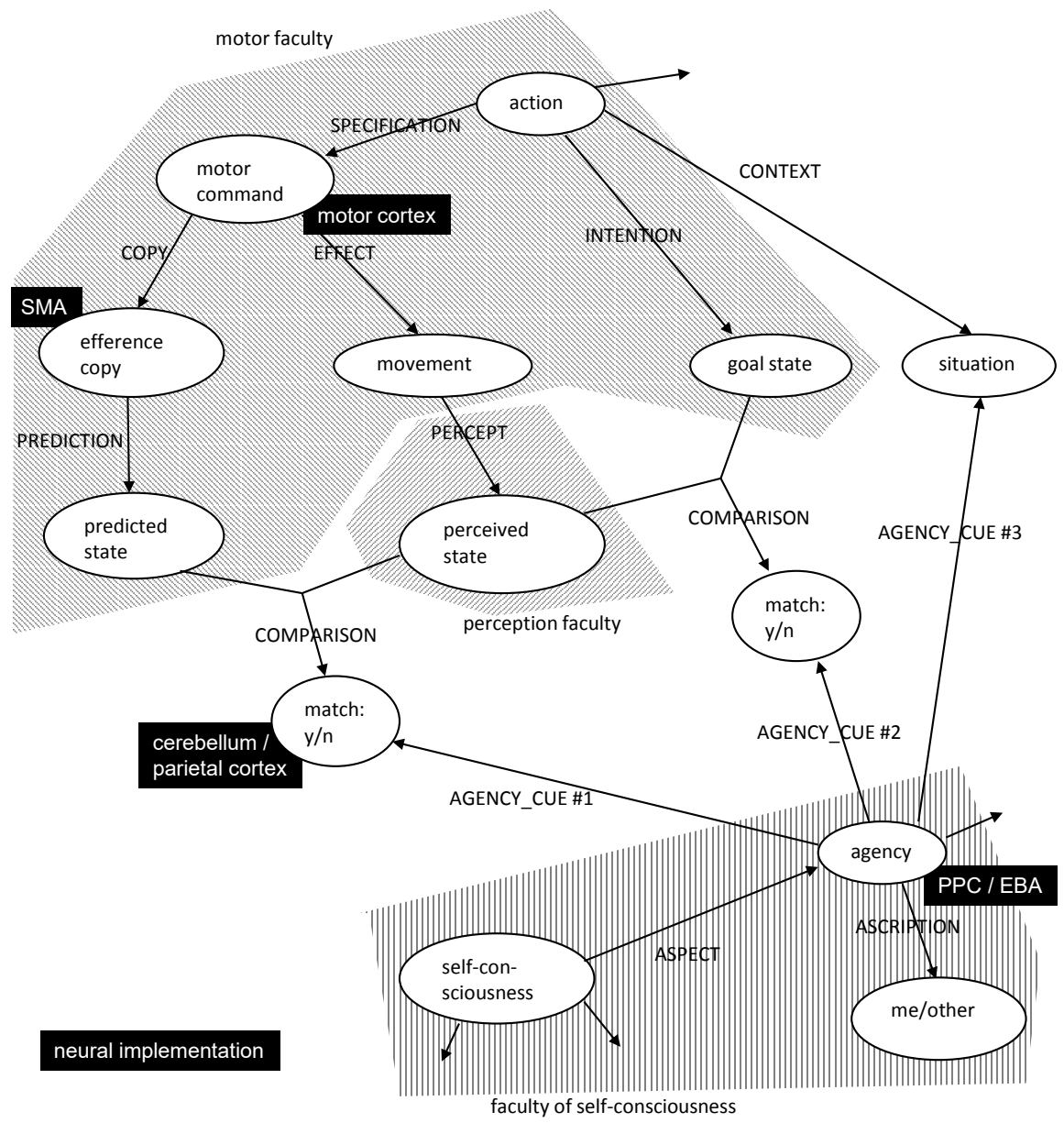

Figure 5: A multifactorial account of agency in frames. Different faculties of the mind are presented by shaded areas. Possible neural implementations are attached to the according nodes as black rectangulars (SMA: supplementary motor area, PPC: posterior parietal cortex, EBA: extrastriate body area).

In Figure 5, a frame is shown that (partly and exemplarily) describes the multifactorial account of agency. For example, a mismatch at the comparator comparing perceived state and goal state serves as one agency cue indicating that I am not the agent of the action (this is the case, for instance, when someone nudges your arm during an arm movement such that you do not reach your goal). 
However, in other situations such a mismatch might not lead you to think that you are not the agent of the action: e.g., perceived state and goal state will not match when you fail to hit the bull's eye as planned when playing darts. Nevertheless, the situation of playing darts and your background knowledge that your skills in darts are not perfect will function as another cue which is, in this situation, weighted more highly than the mismatch. This results in your ascribing the agency to yourself.

Different faculties of the mind (exemplarily) re presented by differently shaded areas since the two-dimensional paper makes an adequate depiction of the different faculties as attributes of a central "person" node very inconvenient. The frame analysis shows how different faculties of the mind interrelate in the production of specific phenomena (agency as an aspect of self-consciousness in this case).

Delusions of control can be described as disturbances in specific values in the frame, for example by unusual values of the comparison outcomes due to deficits in the comparison process or by unusual values of agency ascriptions due to deficits in the weighting process of different cues. At the same time, the frame is able to display the complex effects that disturbances in single nodes can have; e.g., a disturbed value of a COMPARISON node might lead to disturbed values in the ASCRIPTION node in cases where it is not outweighed by other agency-nodes.

This example of analyzing specific phenomena and their pathological disturbances is, moreover, embedded into a general theory of self-consciousness (Vosgerau 2009), such that the general pattern of analysis can be fruitfully applied to other phenomena (e.g., authorship of thoughts or ownership of body parts; Synofzik, Vosgerau \& Newen 2008).

Moreover, the frame makes it possible to integrate data concerning neural circuits. Just to give a few examples: some brain areas are attached to some nodes in the frame; there is some evidence that renders these brain areas plausible candidates for being the neural correlate of the according values in the frame. The motor cortex is likely to be involved in forming and issuing the motor command (Beurze et al. 2010); there is considerable evidence that the supplementary motor area (SMA) plays a crucial role in the generation of a copy of the motor command ("efference copy") that is further used in prediction (Haggard \& Whitford 2004). It has been suggested that the comparison process between predicted state and perceived state may involve the cerebellum and the parietal cortex (Blakemore, Wolpert \& Frith 1999); in addition, it has also been suggested that the poste- 
rior parietal cortex (PPC) and the extrastriate body area (EBA) may participate in the network of areas correlating with the sense of agency (Yomogida et al. 2010). These examples show how experimental data on the neural correlates of the different faculties of the mind can be straightforwardly integrated into the frame analysis, such that a comprehensive picture of the complex interrelations between different faculties of the mind, different disruptions of specific processes, and different brain regions involved in these processes emerges.

\section{Discussion}

Frame theory can be applied to modern operationalized diagnostic criteria of mental disorders, but its use is limited in two ways: $i)$ the mental processes underlying the pathophysiology of mental disorders are not yet sufficiently clarified and $i$ ) frame theory cannot yet render temporal and causal dimensions of mental disorders. Also, the diagnostic structure of some mental disorders is complicated, since such disorders involve a number of functional brain modules, leading to intricate frame structures. During our initial analyses, frequently the question arose as to which functions of the human brain are impaired and how this results in a clinical symptom. As exemplified even by the relatively simple case of phobias, there is an intricate network of interacting brain modules and the pathophysiology of the psychopathologic phenomena proves to be of a very intricate nature. Identifying constraints between attributes in frames for mental disorders will prove to be difficult because in clinical reality there is hardly ever a strict correlation, but rather a certain predisposition or statistical association. Another aspect which needs to be addressed is the question of a continuum between symptoms of mental disorders and "diluted" or less intense similar symptoms in healthy people. This has been analyzed for some major symptoms of mental disorders like delusions and hallucinations, and could indicate that there may be no absolute boundaries between "health" and "disease." This issue is referred to as the "dimensional" nature of mental disorders and one of the central topics of the current revision process of DSM-IV (Brown \& Barlow, 2005). It will be necessary to include such aspects in the Frame Analysis of mental disorders.

With such limitations and challenges at hand, it seems attractive to postpone any further frame analysis of mental disorders until the pathophysiology of the mental disorders to be analyzed is known. However, as the example of Parkin- 
son's disease shows, it seems unlikely that "simple" pathophysiologies will be discovered. Thus, we decided to focus on core features of the pathophysiology and symptomatology of psychotic disorders, in this case the disturbance of the concept of self-agency, to limit the scope of phenomena to be explained by more detailed frame analyses of mental disorders. Two features speak in favor of using frame theory: i) it provides a systematic and well-defined approach which provides general rules usable for all mental disorders as regards their pathophysiology, symptomatology and classification, and ii) it may also be useful for analyzing the historic changes of diagnostic conceptions of mental disorders. Thus, the approach to progress in this area - as exemplified by schizophrenia - needs to be twofold: firstly, it is well worth the effort to analyze historic texts of the early times of the conceptualization of schizophrenia as an example, because these definitions were made without any knowledge about the pathophysiology of the disorder and have developed over time. Secondly, it is necessary to analyze the neurobiological underpinnings of core concepts of mental disorders using frame analyses including time-variability and individual precipitating factors for their disturbances in mental disorders.

\section{Acknowledgement}

This project was supported by a grant from the "Human Sciences Research Center" (Humanwissenschaftlich-Medizinisches Forschungszentrum) of the HeinrichHeine-University Düsseldorf.

\section{References}

American Psychiatric Association. 2000. Diagnostic and Statistical Manual of Mental Disorders, Fourth Edition, Text Revision. Washington: American Psychiatric Association.

Barsalou, L. W. 1992a. Cognitive Psychology. Hillsdale, NJ: Lawrence Erlbaum Associates.

Barsalou, L. W. 1992b. Frames, Concepts, and Conceptual Fields. In A. Lehrer \& E. F. Kittay (eds.), Frames, fields, and contrasts, 21-74. Hillsdale, NJ: Lawrence Erlbaum Associates.

Barsalou, L. W. 1999. Perceptual Symbol Systems. Behavioral and Brain Sciences 22. $577-660$. 


\section{References}

Beurze, S. M., I. Toni, L. Pisella \& W. P. Medendorp 2010. Reference Frames for Reach Planning in Human Parietofrontal Cortex. fournal of Neurophysiology 104. 1736-1745. 10.1152/jn.01044.2009.

Blakemore, S.-J., D. M. Wolpert \& C.D. Frith 1999. The Cerebellum Contributes to Somatosensory Cortical Activity during Self-Produced Tactile Stimulation. NeuroImage 10. 448-459.

Bleuler, E. 1911. Dementia praecox oder die Gruppe der Schizophrenien. Leipzig und Berlin: Verlag Franz Deuticke.

Brown, T. A., \& D. H. Barlow 2005. Dimensional versus categorical classification of mental disorders in the fifth edition of the Diagnostic and Statistical Manual of Mental Disorders and beyond: comment on the special section. Fournal of Abnormal Psychology 114. 551-556.

Calabresi, P., B. Picconi, L. Parnetti \& M. Di Filippo 2006. A convergent model for cognitive dysfunctions in Parkinson's disease: the critical dopamine-acetylcholine synaptic balance. Lancet Neurology 5. 974-983.

Damsa, C., M. Kosel \& J. Moussally 2008. Current status of brain imaging in anxiety disorders. Current Opinion in Psychiatry 22. 96-110.

Haggard, P., Whitford, B. 2004. Supplementary motor area provides an efferent signal for sensory suppression. Cognitive Brain Research 19. 52-58.

Kraepelin, E. 1899. Psychiatrie. Ein Lehrbuch für Studierende und Ärzte. 5th Edition. Leipzig: Barth.

Lees, A. J., J. Hardy \& T. Revesz 2009. Parkinson's disease. Lancet, 373. 2055-2066. López-Muñoz, F., P. García-García, J. Sáiz-Ruiz, J. E. Mezzich, G. Rubio, E. Vieta \& C. Alamo 2008. A bibliometric study of the use of the classification and diagnostic systems in psychiatry over the last 25 years. Psychopathology 41. 214-225.

Munafo, M. R., S. M. Brown \& A. R. Hariri 2008. Serotonin transporter (5-HTTLPR) genotype and amygdala activation: a meta-analysis. Biological Psychiatry 63. 852-857.

Petersen, W. 2015. Representation of Concepts as Frames. In Gamerschlag T., D. Gerland, R. Osswald \& W. Petersen (eds.) Meaning, Frames, and Conceptual Representation, 39-63. Studies in Language and Cognition 2. Düsseldorf: Düsseldorf University Press. (Reprinted from J.; Toccafondi, F. \& Stemberger, G. (eds.): Complex Cognition and Qualitative Science. Volume 2 of The Baltic International Yearbook of Cognition, Logic and Communication, 151-170, Uni- 
versity of Latvia, Riga, 2007.)

Pull, C. B. 2008. Recent trends in the study of specific phobias. Current Opinion in Psychiatry 21. 43-50.

Schneider, K. 1971. Klinische Psychopathologie. 9th Edition. Stuttgart: Thieme Verlag.

Seitz, R. J., Gaebel W., Zielasek J. 2011. Modular networks involving the medial frontal cortex: towards the development of neuropsychiatry. World fournal of Biological Psychiatry. 12 (4). 249-59.

Storch, A., G. Ebersbach, G. Fuchs, W.H. Jost, P. Odin, G. Reifschneider \& M. Bauer 2008. Depression beim idiopathischen Parkinson-Syndrom. Teil 1: Epidemiologie, Pathophysiologie, Klinik und Diagnostik. Fortschritte der Neurologie und Psychiatrie 76. 715-724.

Synofzik, M., G. Vosgerau \& A. Newen 2008. 'I move, therefore I am: A new theoretical framework to investigate agency and ownership'. Consciousness and Cognition 17: 411-424.

Vosgerau, G. 2009. 'Die Stufentheorie des Selbstbewusstseins und ihre Implikationen für das Verständnis psychiatrischer Störungen'. Journal für Philosophie und Psychiatrie 2, 16 pages, http://www.jfpp.org/jfpp-2-2009-02.html.

World Health Organization 1992. The ICD-10 classification of mental and behavioural disorders. Geneva: World Health Organization.

Yomogida, Y., M. Sugiura, Y. Sassa, K. Wakusawa, A. Sekiguchi, A. Fukushima, H. Takeuchi, K. Horie, S. Sato \& R. Kawashima 2010. The neural basis of agency: An fMRI study. NeuroImage 50. 198-207.

Zgaljardic, D. J., N. S. Foldi \& J. C. Borod 2004. Cognitive and behavioral dysfunction in Parkinson's disease: neurochemical and clinicopathological considerations. Fournal of Neural Transmission 111. 1287-1301.

\section{Authors}

Jürgen Zielasek

Heinrich-Heine-University Düsseldorf

Dept. of Psychiatry and Psychotherapy

juergen.zielasek@lvr.de

Gottfried Vosgerau

Heinrich-Heine-University Düsseldorf 


\section{References}

Dept. of Philosophy

vosgerau@phil.hhu.de

Wolfgang Gaebel

Heinrich-Heine-University Düsseldorf

Dept. of Psychiatry and Psychotherapy

wolfgang.gaebel@hhu.de

Karin Fauerbach

Heinrich-Heine-University Düsseldorf

Dept. of Psychiatry and Psychotherapy

karin.fauerbach@lvr.de

Irem Girgin

Heinrich-Heine-University Düsseldorf

Dept. of Psychiatry and Psychotherapy

irem.girgin@lvr.de

Sarah Jungbluth

Heinrich-Heine-University Düsseldorf

Dept. of Psychiatry and Psychotherapy

sarah.jungbluth@lvr.de

Julia Weiland

Heinrich-Heine-University Düsseldorf

Dept. of Psychiatry and Psychotherapy

julia.weiland@lvr.de

Sebastian Löbner

Heinrich-Heine-University Düsseldorf

Departement of Linguistics and Information Science

loebner@phil.hhu.de 
